\title{
Wide heterogeneity of congenital myasthenic syndromes: analysis of clinical experience in a tertiary center
}

\author{
Anna Cho ${ }^{1,2 \oplus}$, Soo Yeon Kim ${ }^{3,4 \oplus}$, Jin Sook Lee ${ }^{5 \oplus}$, Byung Chan Lim ${ }^{3,4 \oplus}$, Hunmin Kim ${ }^{1 \oplus}$, Hee Hwang ${ }^{1 \oplus}$, and Jong-Hee Chae ${ }^{3,4, *}$ \\ ${ }^{1}$ Department of Pediatrics, Seoul National University Bundang Hospital, Seongnam, Korea \\ ${ }^{2}$ Rare Disease Center, Seoul National University Bundang Hospital, Seongnam, Korea \\ ${ }^{3}$ Department of Pediatrics, Seoul National University Children's Hospital, Seoul National University College of Medicine, Seoul, Korea \\ ${ }^{4}$ Rare Disease Center, Seoul National University Hospital, Seoul, Korea \\ ${ }^{5}$ Department of Pediatrics, Gachon University Gil Medical Center, Gachon University College of Medicine, Incheon, Korea
}

\begin{abstract}
Purpose: Congenital myasthenic syndrome (CMS) is a clinically and genetically heterogeneous group of disorders characterized by impaired neuromuscular transmission. This study aims to provide the clue for early diagnosis and improved therapeutic strategies in CMS.

Materials and Methods: Through the targeted panel sequencing including twenty CMS causative genes, eleven patients were genetically confirmed and enrolled in this study. A retrospective medical record review was carried out for the clinical and laboratory data analysis.

Results: The age of patients ranged from 5 to 23 years, with the median age of 16 years. The peak age at onset of symptoms was the neonatal period. Seven out of the eleven patients were symptomatic at birth. The most commonly reported initial finding was generalized hypotonia with poor sucking and crying. Mean time to accurate diagnosis was $9.3 \pm 5.0$ years. Total fifteen different variants in seven genes associated with CMS (DOKT, AGRN, RAPSN, CHRNE, COLQ, SLC5A7, and GFPT1) were identified.

Conclusion: We describe the clinical and genetic characteristics of CMS patients and treatment outcome in a single tertiary center. High clinical suspicion and timely molecular diagnosis is particularly important for the tailored therapy to maximize clinical improvement in CMS.
\end{abstract}

Key words: Congenital myasthenic syndrome, Therapeutic effect, Next generation sequencing

\section{Introduction}

Congenital myasthenic syndrome (CMS) is a clinically and genetically heterogeneous group of disorders characterized by impaired neuromuscular transmission [1-3]. It is a very rare hereditary muscle disease with the prevalence of individual dis- eases reported as 0.1-1/100,000 [4-7]. Clinically, it shows various symptoms such as motor weakness, ptosis, respiratory failure, dysphagia, scoliosis, and joint contractures. It shows a broad spectrum of severity from the case of floppy infant syndrome that develops in the neonatal stage to the case with only mild ptosis in adulthood. Often, it is difficult to distinguish CMS from

Received: 14 October 2020, Revised: 27 October 2020, Accepted: 28 October 2020, Published: 31 December 2020

${ }^{*}$ Corresponding author: Jong-Hee Chae, M.D., Ph.D. (iD https://orcid.org/0000-0002-9162-0138

Division of Pediatric Neurology, Department of Pediatrics, Seoul National University Children's Hospital, Seoul National University College of Medicine, Rare Disease Center, Seoul National University Hospital, 101 Daehak-ro, Jongno-gu, Seoul 03080, Korea.

Tel: +82-2-2072-3622, Fax: +82-2-743-3455, E-mail: chaeped1@snu.ac.kr

Conflict of interest: The authors declare that they do not have any conflicts of interest.

(ac) This is an open-access article distributed under the terms of the Creative Commons Attribution Non-Commercial License (http://creativecommons.org/licenses/by-nc/4.0/) which permits unrestricted non-commercial use, distribution, and reproduction in any medium, provided the original work is properly cited.

(c) Copyright 2020 by the Korean Society of Medical Genetics and Genomics 
other neuromuscular disorders including congenital myopathy, congenital muscular dystrophy, limbs girdle muscular dystrophy, and seronegative myasthenia gravis $[8,9]$.

The causative genes for congenital myasthenia syndrome are very diverse. More than 30 causative genes of CMS have been reported to date and new genes are constantly being discovered $[10,11]$. Commonly identified causative genes of CMS include CHRNE, RAPSN, COLO, DOK7, GFPT1, and CHAT with variations of ethnicity $[4,6,12-14]$. Since next generation sequencing has been increasingly used for the diagnosis of hereditary myopathies, a number of patients with CMS have been genetically diagnosed and receiving drug treatment with remarkable clinical improvement. The importance of genetic diagnosis is particularly emphasized because the response to medical treatment varies depending on the molecular subtypes in CMS $[2,3,10,15]$.

Here, we describe the clinical and genetic characteristics of CMS patients diagnosed and treated in a single tertiary center. Through this, we aim to provide the clue for early diagnosis and improved therapeutic strategies in CMS.

\section{Materials and Methods}

\section{Patients}

This study was approved by Seoul National University Hospital Institutional Review Board (IRB No. H-2009-070-1157). All cases were ascertained from the neuromuscular disorders database in the Seoul National University Children's Hospital. We performed next generation sequencing (NGS) in total 230 patients with genetically unclassified neuromuscular disorders (onset age $\leq 10$ years). Before the NGS testing, genetic tests including spinal muscular atrophy, Duchenne muscular dystrophy, and myotonic dystrophy were performed in some patients, based on the clinical and pathologic findings, but were negative. Eleven patients from nine unrelated Korean families and one Emirati family were included in this study. All cases were genetically confirmed as CMS. A retrospective medical record review was carried out for the clinical data analysis.Diurnal variation and ptosis were subjectively classified to mild, moderate, and severe symptoms. The severity of scoliosis was scaled by Cobb angles (mild: 10-30 degree, moderate: $30-45$ degree, and severe: $>45$ degree). Respiratory dysfunction was described as severe when the patient was dependent on permanent ventilator care or underwent therapeutic tracheostomy. Laboratory data including serum creatine kinase, electrophygiologic studies, and muscle pathology were also reviewed.

\section{Genetic analysis}

DNA was extracted from peripheral blood leukocytes. Informed consent was obtained from all families for the extraction of DNA to perform mutation analysis. We performed targeted panel sequencing of 432 selected genes known to cause monogenic neuromuscular disorders including 20 CMS causative genes (CHRNA1, CHRNB1, CHRND, CHRNE, RAPSN, CHAT, COLO MUSK, DOK7, AGRN, GFPT1, DPAGT1, LAMB2, SCN4A, CHRNG, PLEC, ALG2 ALG14, SYT2 PREPL) [16]. The genetic variants identified were validated using Sanger sequencing and segregation studies were performed using parental DNA samples when needed. The variants identified were considered to be pathogenic or likely pathogenic according to the 2015 American College of Medical Genetics and Genomics guidelines [17].

\section{Results}

We found eleven CMS patients from ten unrelated families (Table 1). The age of patients ranged from 5 to 23 years, with the median age of 16 years. The peak age at onset of symptoms was the neonatal period. Seven out of the eleven patients were symptomatic at birth. The most commonly reported initial finding was generalized hypotonia with poor suck and cry. Diurnal variation in seven cases and mild ptosis in six cases were observed. Respiratory distress at birth was noticed in seven patients, four of them suffered transiently and recovered after the neonatal period. Eight patients showed variable degrees of scoliosis. Mean time to accurate diagnosis was $9.3 \pm 5.0$ years. Total fifteen different variants in seven genes associated with CMS (DOK7, AGRN, RAPSN, CHRNE, COLQ, SLC5A7, and GFPT1) were identified.

\section{Postsynaptic endplate development and maintenance defects (DOK7, AGRN, RAPSN)}

The homozygous c. $1185 \mathrm{C}>\mathrm{G}$ mutations and compound heterozygous c. $467 C>A$ and c. $1502 c>T$ mutations of DOK7 genes were identified in two patients (case 1 and 2). The homozygous c.5023G $>$ A mutations of $A G R N$ were identified in two brothers in one family (case 3-1 and case 3-2). One case of the compound heterozygous c. $133 \mathrm{G}>\mathrm{A}$ and c.690G $>$ mutations of RAPSN were found (case 4). All five patients were symptomatic at birth. Four patients with DOK 7 and $A G R N$ mutations were initially presenting as floppy infant syndrome with a clinical diagnosis of congenital myopathy. One patient (case 1) was able to walk with assist in early childhood, but weakness worsened to the state where she could not stand up on her own at 10 years old. The 


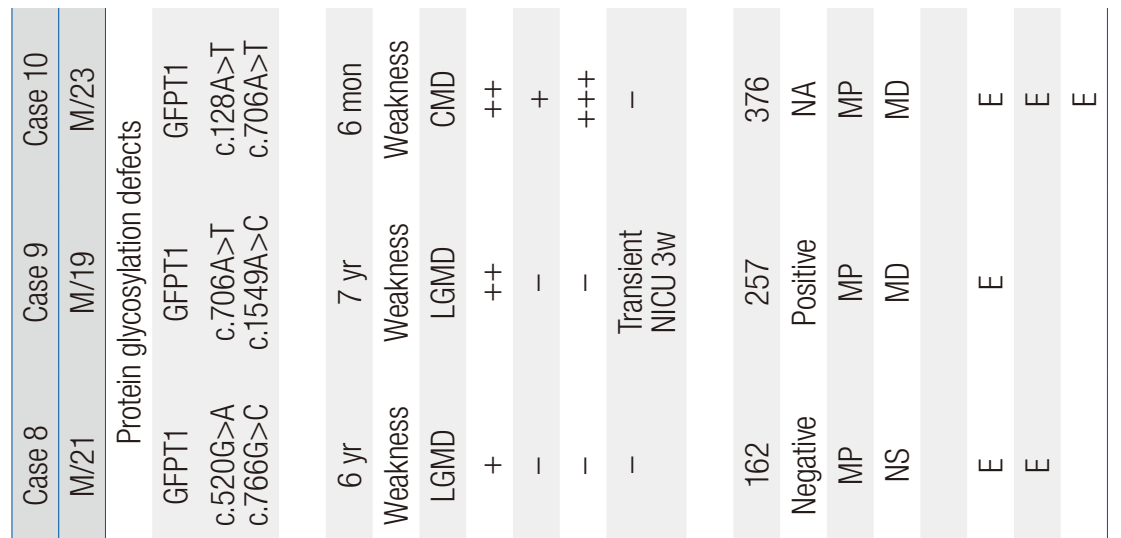

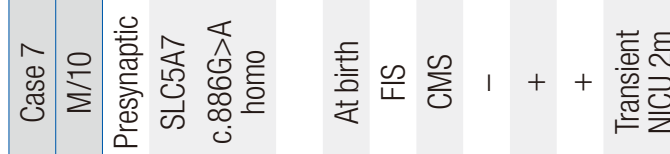

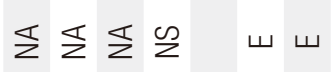

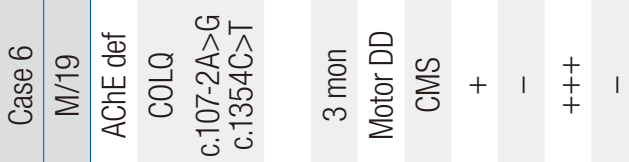

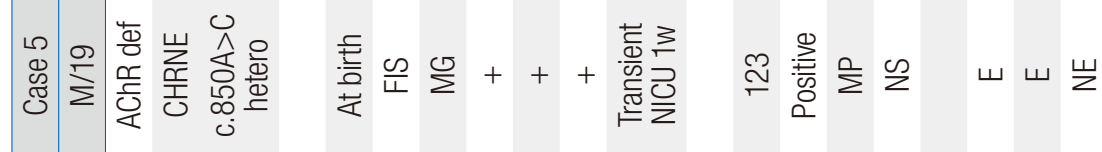

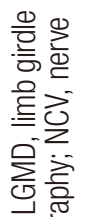

酸产

을

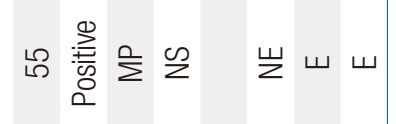

焉焉

营言

$\sum_{0}^{\infty} \frac{1}{0}$

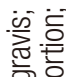

즐 응

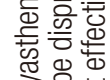

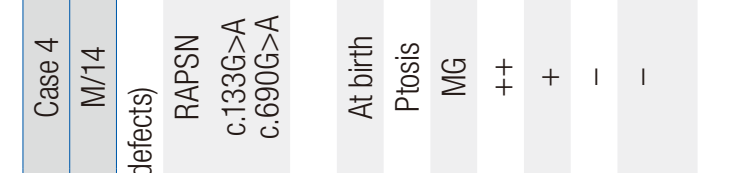

ণ

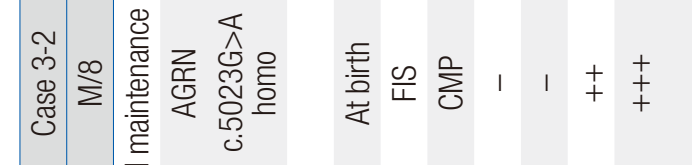

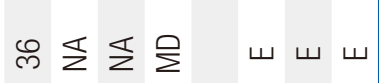

它离剀

돌

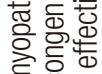

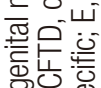

Oे

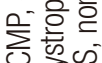

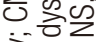

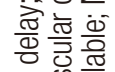

푸에

흥로을

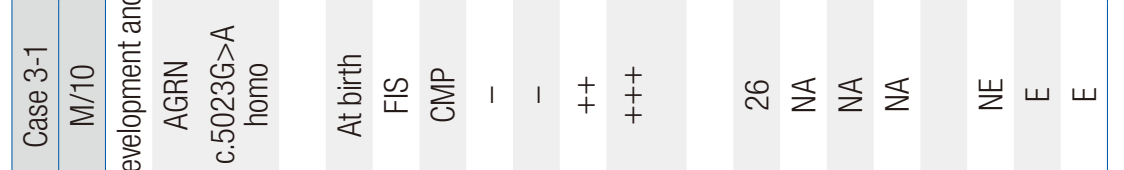

焉

응

हैं

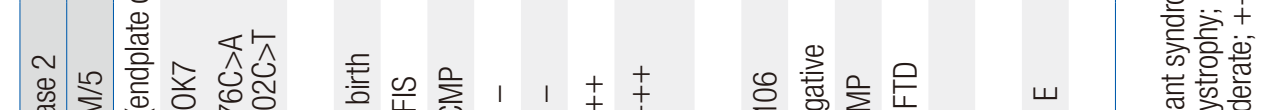

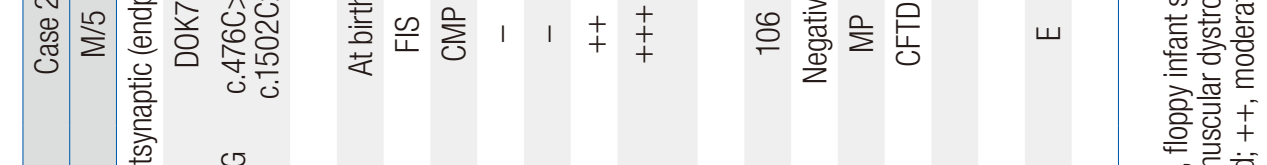

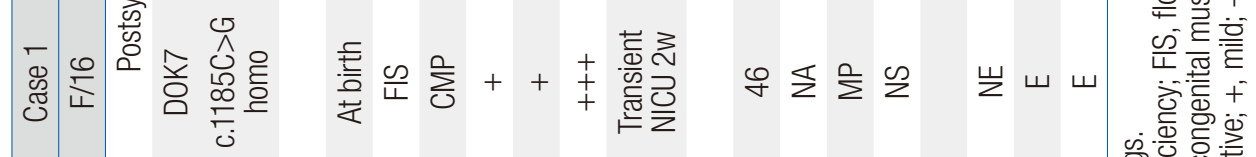

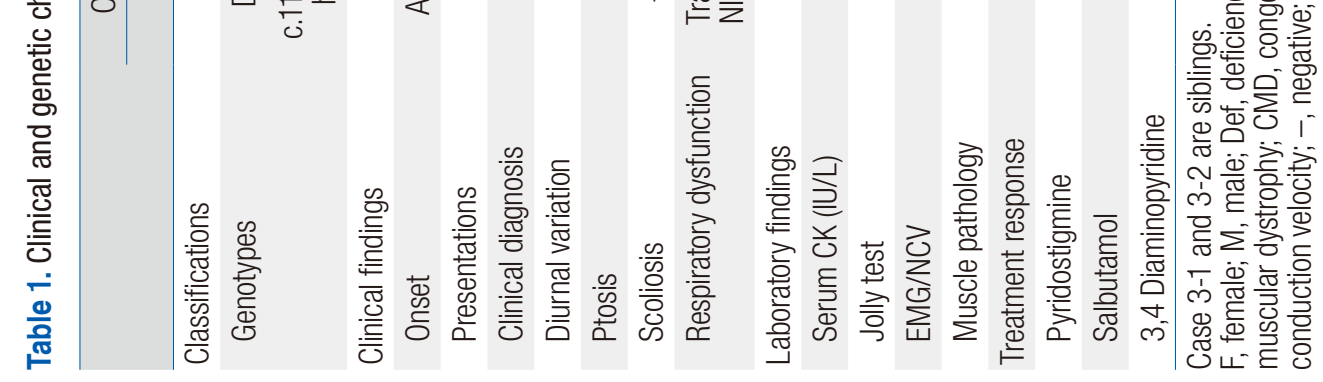


maximal motor performance achieved in others (case 2-4) was rolling over at diagnosis. These three patients (case 2-4) underwent therapeutic tracheostomy for the management of severe respiratory distress. Four patients had moderate to severe scoliosis with a Cobb angle of 30 degree or more and one of them (case 1) underwent surgical correction at her age of 13 years. Pyridostigmine was not effective or somewhat worsen in two patients (case 1 and case 3-1) but partially effective in case 3-2 ( $6 \mathrm{mg} / \mathrm{kg} /$ day). Salbutamol therapy was effective in all patients with DOK 7 and $A G R N$ mutations. Three patients were under the combination therapy of salbutamol (1-2 mg/kg/day) and 3, 4 diaminopyridine (0.5-1 mg/kg/day), showing variable degrees of improvement. At age 15 years, case 1 was able to climbing stairs and independent in her daily activities. Case 2 started to stand with assist at his age of 5 years. Two brothers with AGRN mutations could walk independently (case 3-1) or with assist (case 3-2) since early childhood. Case 4 with RAPSN mutations presented with ptosis at birth. He developed facial weakness and mild motor developmental delay during infancy and was diagnosed as myasthenia gravis with the positive response to neostigmine test at his age of 2 years. The test for acetylcholine receptor (AchR) antibodies was negative but ptosis had been well controlled with oral pyridostigmine ( $6 \mathrm{mg} / \mathrm{kg} /$ day). The molecular diagnosis was made at his age of 11 years.

\section{Postsynaptic acetylcholine receptor deficiency (CHRNE)}

Case 5 harbored a heterozygous c.850A $>$ C mutation of CHRNE. He presented with generalized hypotonia with transient respiratory distress at birth and developed gross motor delay, facial weakness, and ptosis during infancy and childhood. At age 13 years, he reported mild fluctuations of weakness, usually related to exertion, and the electrophysiologic study revealed abnormal electrodecremental response to repetitive nerve stimulation (Jolly test positive). The molecular diagnosis was made at his age of 15 years. Drug treatment with pyridostigmine $(4 \mathrm{mg} / \mathrm{kg} /$ day) and salbutamol $(0.5 \mathrm{mg} / \mathrm{kg} /$ day) resulted in improvement in muscle strength, but 3.4 diaminopyridine was not effective.

\section{Endplate acetylcholinesterase deficiency $(C O L O)$}

Case 6 carried compound heterozygous c.107-2A $>\mathrm{G}$ and c. 1354C>T mutations of COLO. He presented with gross motor delay during early infancy and developed proximal dominant generalized weakness in childhood. There was a diurnal variation, with worsening of weakness in the afternoon. Jolly test was positive but pyridostigmine did not improve muscle weakness.
After the molecular diagnosis was confirmed at his age of 16 years, he showed significant improvement with the combination therapy of salbutamol $(0.2 \mathrm{mg} / \mathrm{kg} /$ day $)$ and 3.4 diaminopyridine (0.5 mg/kg/day).

\section{High-affinity presynaptic choline transporter (SLC5A7)}

The homozygous c.886G >A mutations of SLC5A7 were identified from an Emirati boy (case 7). He had a history of respiratory distress during neonatal period and showed persistent ptosis since birth. He was clinically diagnosed with CMS but previous sequencing result of DOK 7, CHRNE, RAPSN, and CHAT analysis in UK was negative. The NGS based targeted panel analysis revealed SLC5A7 mutations at his age of 5 years. He showed significant improvement with the combination of pyridostigmine $(6 \mathrm{mg} / \mathrm{kg} /$ day $)$ and salbutamol $(0.2 \mathrm{mg} / \mathrm{kg} /$ day $)$ therapy.

\section{Protein glycosylation defects (GFPT1)}

Three different compound heterozygous variants of GFPT1 genes were identified in case $8-10$ (c.520G $>A$ and c.766G $>C$, c.706A $>$ T and c. $1549 \mathrm{~A}>\mathrm{C}$, and c. $128 \mathrm{~A}>\mathrm{T}$ and c.706A $>$ T). All patients presented with a limb girdle muscular dystrophy phenotype having proximal limb but no facial muscle weakness. Case 8 and case 9 began having difficulty walking and climbing stairs in the childhood. Case 10 presented with gross motor delay during infancy and developed proximal limb weakness and severe scoliosis during childhood. The electrophysiologic study revealed chronic myopathic changes in all cases. Serum creatine kinase was mildly elevated and muscle biopsy revealed chronic degenerative process with a pathologic diagnosis of muscular dystrophy in two patients. After molecular diagnosis of CMS, all patients benefited from pyridostigmine therapy and two patients showed additional improvement from salbutamol.

\section{Discussion}

We report detailed clinical and genetic features of eleven cases with CMS. All patients shared the clinical features of muscle weakness, but the age of onset, distribution and severity of weakness, and response to treatment were very diverse according to the genes harboring the mutations. Typical clinical features were generalized muscle weakness presenting in the neonatal period or early infancy. Diurnal variation of weakness was not so much distinct during infantile and early childhood period and often appeared after childhood as an important clinical clue. Presence of diurnal fluctuations should raise clinical suspicion of 
CMS even in patients diagnosed with myopathy previously [8]. Ptosis was recognized in five patients but often combined with facial weakness, leading to difficulty in differentiating it from other hereditary myopathy faces. Seven patients had respiratory distress at birth and three patients underwent tracheostomy for managing the persistent respiratory insufficiency. Eight patients had scoliosis and three of them were treated surgically. Paying attention to the posture of patients and continued assessment of spine throughout lifespan is essential.

Laboratory findings were not very helpful in the differential diagnosis of CMS in our cohort. Two cases showed Jolly test negative and case 6 showed once negative and once positive result on repeated tests. All seven patients who underwent electrodiagnostic tests showed myopathic findings with shortduration and low-amplitude motor unit potentials. This is thought to result from functional loss of myofibers caused by chronic neuromuscular transmission failure [9]. Muscle biopsy was performed in nine patients and showed nonspecific myopathic findings with myofiber size variations and minimal degenerating changes in five cases. However, one patient (case 2) was pathologically diagnosed as a congenital myopathy with type 1 fiber predominance and three patients (case 3-2, case 9 , and case 10) were diagnosed as muscular dystrophies before molecular diagnosis of CMS. Even serum creatine kinase were mildly elevated in two cases with GFPT1 mutations.

The response to treatment depends on the subtype of CMS $[2,3,10,15,18]$. Traditionally, pyridostigmine, a competitive acetylcholinesterase inhibitor, was the mainstay of treatment for the neuromuscular junction disorders. Oral pyridostigmine can be given at an initial dose of 0.5 to $1 \mathrm{mg} / \mathrm{kg}$ every 4 to 6 hours and the maximum recommended total daily dose is $7 \mathrm{mg} / \mathrm{kg}$. Consistent with previous reports, our patients with defects of acetylcholine receptor, presynaptic choline transporter, and protein glycosylation had much benefited from pyridostigmine. But some subtypes such as end plate acetylcholinesterase deficiency due to COLQ mutations and DOK7-related CMS are known to refractory or deteriorate with pyridostigmine. Patients with DOK7 and COLQ mutations in our cohort derived no benefit or worsened with pyridostigmine. Whereas salbutamol were very effective in patients with $D O K 7$ and $C O L O$ deficiency and partially beneficial in other subtypes of CMS. Oral salbutamol can be given at an initial dose of $4 \mathrm{mg} /$ day and titrated up to $12 \mathrm{mg} /$ day. A 3,4-Diaminopyridine (amifampridine; Ruzurgi, available at Korean Orphan \&t Essential Drug Center) is a potassium channel blocker working on the presynaptic nerve terminal [15]. The pediatric starting dose is $0.25-0.5 \mathrm{mg} / \mathrm{kg} /$ day and titrated up to $1 \mathrm{mg} / \mathrm{kg} /$ day total dose if necessary. It was a useful add-on therapy for the patients with mutations of DOK7, AGRN, COLO, and GFPT1 in our experience.

Our study suggested the importance of molecular diagnosis of CMS to warrant appropriate treatment. Due to the wide heterogeneity of clinical and genetic features, the diagnosis of CMS remains challenging even for experienced clinicians. The number of causative genes continues to grow and the clinical differentiation of subtypes is usually very difficult. The advent of NGS technologies opened a new era of molecular genetic diagnosis in various neuromuscular disorders. Since molecular diagnosis is crucial for the therapeutic decision making in CMS, it is important for clinicians to recognize clinical clues and confirm the genetic analysis earlier.

\section{Acknowledgements}

This research was funded by Research program of Korea Centers for Disease Control and Prevention (2018-ER6901-02).

\section{References}

1. Engel AG. Congenital myasthenic syndromes in 2018. Curr Neurol Neurosci Rep 2018;18:46.

2. Rodríguez Cruz PM, Palace J, Beeson D. The neuromuscular junction and wide heterogeneity of congenital myasthenic syndromes. Int J Mol Sci 2018;19:1677.

3. Engel $A G$, Shen $X M$, Selcen $D$, Sine SM. Congenital myasthenic syndromes: pathogenesis, diagnosis, and treatment. Lancet Neurol 2015;14:420-34.

4. Azuma $Y$, Nakata $T$, Tanaka $M$, Shen $X M$, Ito $M$, Iwata $S$, et al. Congenital myasthenic syndrome in Japan: ethnically unique mutations in muscle nicotinic acetylcholine receptor subunits. Neuromuscul Disord 2015;25:60-9.

5. McMacken G, Abicht A, Evangelista T, Spendiff S, Lochmüller H. The increasing genetic and phenotypical diversity of congenital myasthenic syndromes. Neuropediatrics 2017;48:294-308.

6. Natera-de Benito D, Töpf A, Vilchez JJ, González-Quereda L, Domínguez-Carral J, Díaz-Manera J, et al. Molecular characterization of congenital myasthenic syndromes in Spain. Neuromuscul Disord 2017;27:1087-98.

7. Rodriguez Cruz PM, Palace J, Beeson D. Inherited disorders of the neuromuscular junction: an update. J Neurol 2014;261:2234-43.

8. Kao JC, Milone M, Selcen D, Shen XM, Engel AG, Liewluck T. Congenital myasthenic syndromes in adult neurology clinic: a long road to diagnosis and therapy. Neurology 2018;91:e1770-7. 
9. Nicolau S, Kao JC, Liewluck T. Trouble at the junction: when myopathy and myasthenia overlap. Muscle Nerve 2019;60:648-57.

10. Vanhaesebrouck $A E$, Beeson $D$. The congenital myasthenic syndromes: expanding genetic and phenotypic spectrums and refining treatment strategies. Curr Opin Neurol 2019;32:696-703.

11. Bonne G, Rivier F, Hamroun D. The 2019 version of the gene table of neuromuscular disorders (nuclear genome). Neuromuscul Disord 2018;28:1031-63.

12. Durmus $H$, Shen $X M$, Serdaroglu-Oflazer $P$, Kara $B$, Parman-Gulsen $Y$,

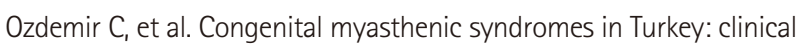
clues and prognosis with long term follow-up. Neuromuscul Disord 2018;28:315-22.

13. Aharoni $S$, Sadeh $M$, Shapira $Y$, Edvardson $S$, Daana M, Dor-Wollman $T$, et al. Congenital myasthenic syndrome in Israel: genetic and clinical characterization. Neuromuscul Disord 2017;27:136-40.

14. Pattrakornkul N, Ittiwut C, Boonsimma P, Boonyapisit K, Khongkhatithum C, Sanmaneechai O, et al. Congenital myasthenic syn- dromes in the Thai population: clinical findings and novel mutations. Neuromuscul Disord 2020;30:851-8.

15. Lee $M$, Beeson $D$, Palace J. Therapeutic strategies for congenital myasthenic syndromes. Ann N Y Acad Sci 2018;1412:129-36.

16. Kaplan JC, Hamroun D. The 2016 version of the gene table of monogenic neuromuscular disorders (nuclear genome). Neuromuscul Disord 2015;25:991-1020.

17. Richards S, Aziz N, Bale S, Bick D, Das S, Gastier-Foster J, et al.; ACMG Laboratory Quality Assurance Committee. Standards and guidelines for the interpretation of sequence variants: a joint consensus recommendation of the American College of Medical Genetics and Genomics and the Association for Molecular Pathology. Genet Med 2015;17:405-24.

18. Farmakidis C, Pasnoor M, Barohn RJ, Dimachkie MM. Congenital myasthenic syndromes: a clinical and treatment approach. Curr Treat Options Neurol 2018;20:36. 